\title{
ANDES

\section{Trastornos de conducta alimentaria grave en adolescentes durante la pandemia COVID-19: un llamado a la acción}

\author{
Severe eating disorders in adolescents during the COVID-19 pandemic: a call to action
}

\author{
Franco Diaz R. ${ }^{\mathrm{a}, \mathrm{b}, \mathrm{c}}$, Lorena Pilicita S. ${ }^{\mathrm{b}}$, Luisana Godoy V.d, Alejandro Donoso F. ${ }^{\mathrm{b}}$ \\ a Unidad de Paciente Crítico, Hospital El Carmen Maipú. Santiago. Chile. \\ bUnidad de Paciente Crítico, Hospital Clínico Dra. Eloísa Díaz I. La Florida. Santiago. Chile. \\ 'Escuela de Medicina, Universidad Finis Terrae. Santiago. Chile. \\ dEscuela de Medicina, Universidad Diego Portales. Santiago. Chile.
}

\section{Sr. Editor,}

Hemos leído con gran interés la editorial en Andes Pediátrica de la Dra. Amezquita ${ }^{1}$, en la que aborda las particularidades de la fisiopatología del SARS-CoV-2 en pacientes obesos. Además, expone cómo el confinamiento ha afectado el estilo de vida y el bienestar psicosocial de niños, niñas y adolescentes (NNA). Si bien la malnutrición por exceso en población pediátrica es alarmante, las medidas de distanciamiento social han producido un efecto inconmensurable en su salud mental y por ende en el desarrollo de trastornos de conducta alimentaria (TCA).

Durante el segundo trimestre del año 2021 ingresamos 3 adolescentes a la unidad de paciente crítico pediátrico (UPCP) diagnosticadas con anorexia nerviosa (AN). Las características clínico-demográficas de las pacientes se resumen en la tabla.

Las pacientes presentaban un buen rendimiento escolar y adecuado entorno social previo a la pandemia, pero desde marzo del año 2020 tuvieron un cambio a modalidad de clases en línea. Dos de ellas mostraron aislamiento social restrictivo, no tuvieron contacto con otros adolescentes ni niños durante el año siguiente y también ausencia de actividades al aire libre ( 1 o 2 ho- ras a la semana en forma irregular). La tercera paciente refiere que luego de un año en estas condiciones volvió a clases en forma semipresencial en 2021 y aumentó su actividad deportiva asociado a restricción calórica. Los síntomas fueron larvados, con una duración mayor a 6 meses en los dos primeros casos, y 4 meses en el último, desde que fueron advertidos por sus familiares. Las pacientes fueron evaluadas en atención primaria y recibieron atención por psicóloga y nutricionista, pero no por psiquiatra. Como gatillante se reportó duelo familiar en uno de los casos.

Todas las pacientes presentaban a su ingreso desnutrición calórico-proteica aguda y deshidratación, una de ellas grave después de varios días de ingesta de mínima de líquidos. El manejo agudo de los casos escapa del objetivo de esta carta, pero es importante señalar que todos presentaban disfunciones orgánicas relacionadas a la baja de peso y restricción de la ingesta calórica-proteica y otros nutrientes. Luego de una adecuada corrección hidroelectrolítica y un aporte progresivo de calorías, las pacientes fueron derivadas a la unidad de adolescencia para continuar con su terapia.

Todos estos factores inciden negativamente en la salud mental, pero lamentablemente este aspecto ha sido desatendido en los NNA durante la pandemia,

Correspondencia:

Franco Diaz R.

francodiazr@gmail.com 
a pesar de los llamados de distintas organizaciones a efectuar intervenciones drásticas en los programas de salud $^{2}$. Los TCA son frecuentemente subvalorados dentro del espectro de los trastornos mentales, probablemente por factores socioculturales y falta de estudios de prevalencia, incidencia, como también intervenciones preventivas, de tamizaje y terapéuticas efectivas. Los síntomas iniciales de TCA son inespecíficos y su intensidad aumenta lentamente. No es de extrañar que los casos presentados hayan sido diagnosticados durante el segundo año de la pandemia, y con un retraso de varios meses de evolución. Del mismo modo, la gravedad es difícil de evaluar, ya que los síntomas y signos son fácilmente ocultados, y los cambios de conductas pueden atribuirse a factores propios del confinamiento.

Las investigaciones sobre las repercusiones de la pandemia COVID-19 en los adolescentes con TCA es escasa, no obstante, estos son uno de los desórdenes psiquiátricos de más difícil tratamiento, aún en condiciones de normalidad. Durante el primer año de la pandemia hubo un reporte de un incremento hasta en un $30 \%$, de las admisiones hospitalarias por $\mathrm{AN}$, con un predominio en adolescentes mujeres ${ }^{3-6}$. Estos datos incipientes muestran solo la punta del iceberg, siendo los problemas de salud mental una de las principales secuelas de la pandemia COVID-198.

Recientemente, Corral et al. ${ }^{7}$ en un estudio retrospectivo de 10 años, encontraron 93 casos de hospitalización por TCA en un servicio de salud mental pediátrico en Santiago de Chile, siendo la AN el más común y con alta comorbilidad de enfermedad psiquiátrica y disfunción familiar. Globalmente, las hospitalizaciones por TCA han aumentado en la última década, pero la hospitalización en UPCP es infrecuente ${ }^{8}$. No obstante, la AN grave tiene una mortalidad entre 3-5\%, un tercio de ellas por suicidio y el resto por alteraciones electrolíticas graves y falla cardiaca refractaria.

Durante la pandemia COVID-19 los individuos con, o en recuperación, de un TCA pueden haber exacerbado sus síntomas por interrupción de sus actividades diarias, cierre prolongado de colegios, aislamiento social, uso de internet, modificaciones de su hábito de sueño y actividad física, stress, ansiedad por la salud y temor al contagio ${ }^{2}$. Además, existe un menor acceso, y más restringido, al soporte social y cuidado médico para una población especialmente necesitada de una atención sanitaria presencial y personalizada.

Los casos aquí comunicados muestran TCA graves
Tabla. Características clínico-demográficas de casos de anorexia nerviosa grave ingresadas en el segundo año de la pandemia COVID-19

\begin{tabular}{llll}
\hline & Caso 1 & Caso 2 & Caso 3 \\
\hline Edad (años) & 13 & 14 & 17 \\
Talla (metros) & 1,61 & 1,59 & 1,66 \\
Antecedentes & $(-)$ & $\begin{array}{l}\text { Duelo } \\
\text { familiar }\end{array}$ & $(-)$ \\
& & 3er trimestre & $1^{\text {er }}$ trimestre \\
Inicio de sintomas & 2020 trimestre & 2020 & 2021 \\
& & & \\
Estado nutricional basal & & 52 & 67 \\
Peso (kg) & 54 & 20.6 & 24.3 \\
IMC (kg/m²) & 20.8 & 0,39 & 0,88 \\
Z-score & 0,65 & 65 & 81 \\
Percentil (\%) & 74 & 6 meses & 4 meses \\
Control ambulatorio & 3 meses & & \\
Estado nutricional ingreso UPCP & 36 & 31,5 & 53,3 \\
Peso (kg) & 13.9 & 12,3 & 19,3 \\
IMC (kg/m²) & $-2,67$ & -4.6 & $-0,56$ \\
Z-score & $<1$ & $<1$ & 29 \\
Percentil (\%) & $33 \%$ & $39 \%$ & $20 \%$ \\
Disminución de peso & Alterada & Alterada & Alterada \\
Función cardiaca & Normal & Alterada & Nornal \\
Función renal & 5 días & 4 días & 4 días \\
Días en UPCP & & & \\
\hline IMC: índice de masa corporal; UPCP: Unidad de Paciente Crítico Pediá- \\
trico.
\end{tabular}

en adolescentes, un grupo especialmente vulnerable durante las crisis sociales, económicas y de salud, como lo ha sido la pandemia COVID-19. Todos los profesionales de la salud están llamados a utilizar herramientas de selección para definir a los NNA en riesgo y derivarlos oportunamente para una evaluación especializada y terapia ${ }^{8}$. Es urgente la necesidad de intervenciones para apoyar a esta población mediante el refuerzo de políticas públicas enfocadas en la salud mental de los NNA durante la pandemia como también en la era post-pandémica, ya que está sindicada como una limitante para el desarrollo de las comunidades y países, afectando en mayor medida a población de bajos ingresos y con un acceso desigual a la salud. 


\section{Referencias}

1. Amezquita MV. El impacto de COVID-19 en la obesidad pediátrica. Andes pediatr. 2021;92(4):501-2.

2. Racine N, McArthur BA, Cooke JE, Eirich R, Zhu J, Madigan S. Global Prevalence of Depressive and Anxiety Symptoms in Children and Adolescents During COVID-19. Jama Pediatr. 2021;175:114250.

3. Branley-Bell D, Talbot CV. Exploring the impact of the COVID-19 pandemic and UK lockdown on individuals with experience of eating disorders. J Eat Disord. 2020;8(1):44.
4. Haripersad YV, Kannegiesser-Bailey $\mathrm{M}$, Morton K, et al. Outbreak of anorexia nervosa admissions during the COVID-19 pandemic. Arch Dis Child. 2021;106(3):e15.

5. Epic Health Research Network. Increasze in adolescent hospitalizations related to eating disorders. Published April 29, 2021. Accedido 15 noviembre 2021. https://ehrnprd.blob.core.windows.net/ wordpress/pdfs/increase-inadolescenthospitalizations related-to-eating disorders.pdf.

6. Dey T, Mansell ZJ, Ranu J. Effect of the COVID-19 Pandemic on Adolescents With Eating Disorders.
JAMA Pediatr. 2021 Published online November 15, 2021. doi:10.1001/ jamapediatrics.2021.4684

7. Corral A, Espinoza V, Yohannessen K, Loyola P, Balboa P, Torrejón C. Trastornos de la alimentación en pacientes hospitalizados en un Servicio de Salud Mental Pediátrico. Andes Pediatr. 2019;90(3):302-8.

8. Losada AV, Marmo J. Herramientas de Evaluación En Trastornos de La Conducta Alimentaria. Madrid: Editorial Académica Española, 2013. Disponible en https://www.aacademica.org/ analia.veronica.losada/14. Accedido 17 septiembre 2021. 